\title{
Rational Fuzzy Cone Contractions on Fuzzy Cone Metric Spaces with an Application to Fredholm Integral Equations
}

\author{
Saif Ur Rehman ${ }^{1}{ }^{1}$ and Hassen Aydi $\mathbb{D}^{2,3,4}$ \\ ${ }^{1}$ Department of Mathematics, Gomal University, Dera Ismail Khan 29050, Pakistan \\ ${ }^{2}$ Institut Supérieur d'Informatique et des Techniques de Communication, Université de Sousse, H. Sousse, Tunisia \\ ${ }^{3}$ Department of Mathematics and Applied Mathematics, Sefako Makgatho Health Sciences University, Ga-Rankuwa, South Africa \\ ${ }^{4}$ China Medical University Hospital, China Medical University, Taichung, 40402, Taiwan
}

Correspondence should be addressed to Hassen Aydi; hassen.aydi@isima.rnu.tn

Received 28 February 2021; Accepted 22 April 2021; Published 10 May 2021

Academic Editor: Liliana Guran

Copyright (c) 2021 Saif Ur Rehman and Hassen Aydi. This is an open access article distributed under the Creative Commons Attribution License, which permits unrestricted use, distribution, and reproduction in any medium, provided the original work is properly cited.

\begin{abstract}
This paper is aimed at proving some common fixed point theorems for mappings involving generalized rational-type fuzzy conecontraction conditions in fuzzy cone metric spaces. Some illustrative examples are presented to support our work. Moreover, as an application, we ensure the existence of a common solution of the Fredholm integral equations: $\mu(\tau)=\int_{0}^{\tau} \Gamma(\tau, v, \mu(v)) d v$ and $v(\tau)$ $=\int_{0}^{\tau} \Gamma(\tau, v, v(v)) d v$, for all $\mu \in U, v \in[0, \eta]$, and $0<\eta \in \mathbb{R}$, where $U=C([0, \eta], \mathbb{R})$ is the space of all $\mathbb{R}$-valued continuous functions on the interval $[0, \eta]$ and $\Gamma:[0, \eta] \times[0, \eta] \times \mathbb{R} \longrightarrow \mathbb{R}$.
\end{abstract}

\section{Introduction}

In 1922, Banach [1] proved a "Banach contraction principle," which is stated as follows: "A self-mapping on a complete metric space verifying the contraction condition has a unique fixed point." This principle plays a very important role in the fixed point theory. A number of researches have generalized it in many directions for single-valued and multivalued mappings in the context of metric spaces. Some of the findings can be found in [2-13] and the references therein. Currently, the fixed point theory is one of the most interested research areas in the field of mathematics. In the last decades, it has been investigated in many fields, such as game theory, graph theory, economics, computer sciences, and engineering.

The theory of fuzzy sets was introduced by Zadeh [14], while the concept of a fuzzy metric space (FM space) was given by Kramosil and Michalek [15]. After that, the stronger form of the metric fuzziness was presented by George and Veeramani in [16]. Later on, in [17], Gregori and Sapena proved some contractive-type fixed point results in complete FM spaces. Some more fixed point results in FM spaces can be found in [18-27] and the references therein.
Initially, in 2007, the concept of a cone metric space was reintroduced by Huang and Zhang [28]. They proved some nonlinear contractive-type fixed point results in cone metric spaces. After the publication of this article, a number of researchers have contributed their ideas in cone metric spaces. Some of such works can be found in [29-34] and the references therein.

In 2015, the basic concept of a fuzzy cone metric space (FCM space) was given by Öner et al. [35]. They presented some key attributes and a "fuzzy cone Banach contraction theorem" in FCM spaces. Later, Rehman and Li [36] extended and improved a "fuzzy cone Banach contraction theorem" and proved some generalized fixed point theorems in FCM spaces. Some more properties and related fixed point results can be found in [37-47].

The aim of this research work is to establish some rational-type fuzzy cone-contraction results in FCM spaces. We use the concept of $[36,39]$ and prove some common fixed theorems under generalized rational-type fuzzy conecontraction conditions in FCM spaces. Some illustrative examples are presented. In the last section, we give an application of two Fredholm integral equations (FIEs). 


\section{Preliminaries}

Definition 1 [47]. An operation $*:[0,1]^{2} \longrightarrow[0,1]$ is called a continuous $t$-norm if

(i) $*$ is commutative, associative, and continuous

(ii) $1 * \eta_{1}=\eta_{1}$ and $\eta_{1} * \eta_{2} \leq \eta_{3} * \eta_{4}$, whenever $\eta_{1} \leq \eta_{3}$ and $\eta_{2} \leq \eta_{4}$, for all $\eta_{1}, \eta_{2}, \eta_{3}, \eta_{4} \in[0,1]$

The basic $t$-norms: the minimum, the product, and the Lukasiewicz continuous $t$-norms are defined by [47]

$$
\begin{gathered}
\eta_{1} * \eta_{2}=\min \left\{\eta_{1}, \eta_{2}\right\} \eta_{1} * \eta_{2}=\eta_{1} \eta_{2}, \\
\eta_{1} * \eta_{2}=\max \left\{\eta_{1}+\eta_{2}-1,0\right\}
\end{gathered}
$$

Definition 2 [35]. A 3-tuple $\left(U, M_{r}, *\right)$ is said to be a FCM space if $P$ is a cone of $E, U$ is an arbitrary set, $*$ is a continuous $t$-norm, and $M_{r}$ is a fuzzy set on $U^{2} \times$ int $(P)$ satisfying the following conditions:

(1) $\forall_{v_{1}, v_{2} \in U} ; M_{r}\left(v_{1}, v_{2}, t\right)>0$ and $M_{r}\left(v_{1}, v_{2}, t\right)=1 \Leftrightarrow v_{1}$ $=v_{2}$

(2) $\forall_{v_{1}, v_{2} \in U} ; M_{r}\left(v_{1}, v_{2}, t\right)=M_{r}\left(v_{2}, v_{1}, t\right)$

(3) $\forall_{v_{1}, v_{2}, v_{3} \in U} ; M_{r}\left(v_{1}, v_{2}, t\right) * M_{r}\left(v_{2}, v_{3}, s\right) \leq M_{r}\left(v_{1}, v_{3}\right.$, $t+s)$

(4) $\forall_{v_{1}, v_{2} \in U} ; M_{r}\left(\nu_{1}, v_{2},.\right)$ : int $(P) \longrightarrow[0,1]$ continuous

for all $t, s \in \sin \operatorname{int}(P)$.

Definition 3 [35]. Let $\left(U, M_{r}, *\right)$ be a FCM space and $v_{1} \in U$ and $\left(v_{j}\right)$ be a sequence in $U$.

(i) $\left(v_{j}\right)$ converges to $v_{1}$ if for $c \in(0,1)$ and $t \gg \theta$ there is $j_{1} \in N$ such that $M_{r}\left(v_{j}, v_{1}, t\right)>1-c$, for $j \geq j_{1}$. We may write this $\lim _{j \longrightarrow \infty} v_{j}=v_{1}$ or $v_{j} \longrightarrow v_{1}$ as $j \longrightarrow \infty$

(ii) $\left(v_{j}\right)$ is Cauchy if for $c \in(0,1)$ and $t \gg \theta$ there is $j_{1}$ $\in N$ such that $M_{r}\left(v_{j}, v_{k}, t\right)>1-c$, for $j, k \geq j_{1}$

(iii) $\left(U, M_{r}, *\right)$ is complete if every Cauchy sequence is convergent in $U$

(iv) $\left(v_{j}\right)$ is fuzzy cone contractive if there is $a \in(0,1)$ so that

$\frac{1}{M_{r}\left(v_{j}, v_{j+1}, t\right)}-1 \leq a\left(\frac{1}{M_{r}\left(v_{j-1}, v_{j}, t\right)}-1\right), \quad$ for $t \gg \theta, j \geq 1$.

Lemma 4 [35]. Let $\left(U, M_{r}\right.$, $)$ be a FCM space and let $\left(v_{j}\right)$ be sequence in $U$ converging to a point $v_{1} \in U$ iff $M_{r}\left(v_{j}, v_{1}, t\right)$ $\longrightarrow 1$ as $j \longrightarrow \infty$ for each $t \gg \theta$.
Definition 5 [36]. Let $\left(U, M_{r}, *\right)$ be a FCM space. The fuzzy cone metric $M_{r}$ is triangular if

$$
\begin{aligned}
\frac{1}{M_{r}\left(v_{1}, v_{3}, t\right)}-1 \leq & \left(\frac{1}{M_{r}\left(v_{1}, v_{2}, t\right)}-1\right) \\
& +\left(\frac{1}{M_{r}\left(v_{2}, v_{3}, t\right)}-1\right), \quad \forall v_{1}, v_{2}, v_{3} \in U, t \gg \theta
\end{aligned}
$$

Definition 6 [35]. Let $\left(U, M_{r}, *\right)$ be a FCM space and $\ell: U$ $\longrightarrow U$. Then, $\ell$ is said to be fuzzy cone contractive if there is $a \in(0,1)$ such that

$\frac{1}{M_{r}\left(\ell v_{1}, \ell v_{2}, t\right)}-1 \leq a\left(\frac{1}{M_{r}\left(v_{1}, v_{2}, t\right)}-1\right), \quad \forall v_{1}, v_{2} \in U, t \gg \theta$

A "fuzzy cone Banach contraction theorem" [35] is stated as follows: "Let $\left(U, M_{r}, *\right)$ be a complete FCM space in which fuzzy cone contractive sequences are Cauchy and $\ell: U \longrightarrow$ $U$ be a fuzzy cone contractive mapping. Then, $\ell$ has a unique fixed point."

In this paper, we present some rational-type fuzzy conecontraction theorems in FCM spaces by using the concept of $[36,39]$. Namely, we prove some common fixed theorems under generalized rational-type fuzzy cone-contraction conditions in FCM spaces without the assumption that the fuzzy cone contractive sequences are Cauchy. We use "the triangular property of the fuzzy cone metric." We also present some illustrative examples to support our work. In the last section, an application of Fredholm integral equations is provided.

\section{Main Results}

In this section, we prove some common fixed point theorems via generalized rational-type fuzzy cone-contraction conditions in FCM spaces.

Theorem 7. Let $\left(U, M_{r}, *\right)$ be a complete FCM space in which $M_{r}$ is triangular. Let $\ell, \hbar: U \longrightarrow U$ be a pair of self-mappings so that

$$
\begin{aligned}
& \frac{1}{M_{r}(\ell \mu, \hbar v, t)}-1 \\
& \leq a\left(\frac{1}{M_{r}(\mu, v, t)}-1\right) \\
& \quad+b\left(\frac{M_{r}(\mu, v, t)}{M_{r}(\mu, \hbar v, 2 t) * M_{r}(\nu, \ell \mu, 2 t)}-1\right) \\
& \quad+c\left(\frac{M_{r}(\mu, \ell \mu, t) * M_{r}(\nu, \hbar v, t)}{M_{r}(\mu, v, t) * M_{r}(\mu, \hbar v, 2 t) * M_{r}(\nu, \ell \mu, 2 t)}-1\right) \\
& \quad+d\left(\frac{1}{M_{r}(\mu, \ell \mu, t)}-1+\frac{1}{M_{r}(\nu, \hbar v, t)}-1\right),
\end{aligned}
$$


for all $\mu, v \in U, t \gg \theta, a \in(0,1)$, and $b, c, d \geq 0$ with $a+$ $b+c+2 d<1$. Then, $\ell$ and $\hbar$ have a common fixed point in $U$.

Proof. Fix $\mu_{0} \in U$ and construct a sequence of points in $U$ such that

$$
\begin{gathered}
\mu_{2 j+1}=\ell \mu_{2 j}, \\
\mu_{2 j+2}=\hbar \mu_{2 j+1}, \\
j \geq 0 .
\end{gathered}
$$

Then, by (5), for $t \gg \theta$,

$$
\begin{aligned}
& \frac{1}{M_{r}\left(\mu_{2 j+1}, \mu_{2 j+2}, t\right)}-1 \\
& =\frac{1}{M_{r}\left(\ell \mu_{2 j}, \hbar \mu_{2 j+1}, t\right)}-1 \\
& \leq a\left(\frac{1}{M_{r}\left(\mu_{2 j}, \mu_{2 j+1}, t\right)}-1\right) \\
& +b\left(\frac{M_{r}\left(\mu_{2 j}, \mu_{2 j+1}, t\right)}{M_{r}\left(\mu_{2 j}, \hbar \mu_{2 j+1}, 2 t\right) * M_{r}\left(\mu_{2 j+1}, \ell \mu_{2 j}, 2 t\right)}-1\right) \\
& +c\left(\frac{M_{r}\left(\mu_{2 j} \ell \mu_{2 j}, t\right) * M_{r}\left(\mu_{2 j+1}, \hbar \mu_{2 j+1}, t\right)}{M_{r}\left(\mu_{2 j}, \mu_{2 j+1}, t\right) * M_{r}\left(\mu_{2 j}, \hbar \mu_{2 j+1}, 2 t\right) * M_{r}\left(\mu_{2 j+1}, \ell \mu_{2 j}, 2 t\right)}-1\right) \\
& +d\left(\frac{1}{M_{r}\left(\mu_{2 j}, \ell \mu_{2 j}, t\right)}-1+\frac{1}{M_{r}\left(\mu_{2 j+1}, \hbar \mu_{2 j+1}, t\right)}-1\right) \\
& =a\left(\frac{1}{M_{r}\left(\mu_{2 j}, \mu_{2 j+1}, t\right)}-1\right) \\
& +b\left(\frac{M_{r}\left(\mu_{2 j}, \mu_{2 j+1}, t\right)}{M_{r}\left(\mu_{2 j}, \mu_{2 j+2}, 2 t\right) * M_{r}\left(x_{2 j+1}, x_{2 j+1}, 2 t\right)}-1\right) \\
& +c\left(\frac{M_{r}\left(\mu_{2 j}, \mu_{2 j+1}, t\right) * M_{r}\left(\mu_{2 j+1}, \mu_{2 j+2}, t\right)}{M_{r}\left(\mu_{2 j}, \mu_{2 j+1}, t\right) * M_{r}\left(\mu_{2 j}, \mu_{2 j+2}, 2 t\right) * M_{r}\left(\mu_{2 j+1}, \mu_{2 j+1}, 2 t\right)}-1\right) \\
& +d\left(\frac{1}{M_{r}\left(\mu_{2 j}, \mu_{2 j+1}, t\right)}-1+\frac{1}{M_{r}\left(\mu_{2 j+1}, \mu_{2 j+2}, t\right)}-1\right) \\
& =a\left(\frac{1}{M_{r}\left(\mu_{2 j}, \mu_{2 j+1}, t\right)}-1\right)+b\left(\frac{M_{r}\left(\mu_{2 j}, \mu_{2 j+1}, t\right)}{M_{r}\left(\mu_{2 j}, \mu_{2 j+2}, 2 t\right)}-1\right) \\
& +c\left(\frac{M_{r}\left(\mu_{2 j+1}, \mu_{2 j+2}, t\right)}{M_{r}\left(\mu_{2 j}, \mu_{2 j+2}, 2 t\right)}-1\right) \\
& +d\left(\frac{1}{M_{r}\left(\mu_{2 j}, \mu_{2 j+1}, t\right)}-1+\frac{1}{M_{r}\left(\mu_{2 j+1}, \mu_{2 j+2}, t\right)}-1\right)
\end{aligned}
$$

By Definition 2 (3), $M_{r}\left(\mu_{2 j}, \mu_{2 j+2}, 2 t\right) \geq M_{r}\left(\mu_{2 j}, \mu_{2 j+1}, t\right)$ $* M_{r}\left(\mu_{2 j+1}, \mu_{2 j+2}, t\right)$, for $t \gg \theta$. One writes

$$
\begin{aligned}
& \frac{1}{M_{r}\left(\mu_{2 j+1}, \mu_{2 j+2}, t\right)}-1 \\
& \leq a\left(\frac{1}{M_{r}\left(\mu_{2 j}, \mu_{2 j+1}, t\right)}-1\right) \\
& \quad+b\left(\frac{M_{r}\left(\mu_{2 j}, \mu_{2 j+1}, t\right)}{M_{r}\left(\mu_{2 j}, \mu_{2 j+1}, t\right) * M_{r}\left(\mu_{2 j+1}, \mu_{2 j+2}, t\right)}-1\right) \\
& \quad+c\left(\frac{M_{r}\left(\mu_{2 j+1}, \mu_{2 j+2}, t\right)}{M_{r}\left(\mu_{2 j}, \mu_{2 j+1}, t\right) * M_{r}\left(\mu_{2 j+1}, \mu_{2 j+2}, 2 t\right)}-1\right) \\
& \quad+d\left(\frac{1}{M_{r}\left(\mu_{2 j}, \mu_{2 j+1}, t\right)}-1+\frac{1}{M_{r}\left(\mu_{2 j+1}, \mu_{2 j+2}, t\right)}-1\right)
\end{aligned}
$$

After simplification, we get that

$$
\frac{1}{M_{r}\left(\mu_{2 j+1}, \mu_{2 j+2}, t\right)}-1 \leq \gamma\left(\frac{1}{M_{r}\left(\mu_{2 j}, \mu_{2 j+1}, t\right)}-1\right), \quad \text { for } t \gg \theta,
$$

where $\gamma=(a+c+d) /(1-b-d)<1$ since $(a+b+c+2 d$ )$<1$. Similarly,

$$
\begin{aligned}
& \frac{1}{M_{r}\left(\mu_{2 j+2}, \mu_{2 j+3}, t\right)}-1 \\
& =\frac{1}{M_{r}\left(\ell \mu_{2 j+2}, \hbar \mu_{2 j+1}, t\right)}-1 \\
& \leq a\left(\frac{1}{M_{r}\left(\mu_{2 j+1}, \mu_{2 j+2}, t\right)}-1\right) \\
& +b\left(\frac{M_{r}\left(\mu_{2 j+1}, \mu_{2 j+2}, t\right)}{M_{r}\left(\mu_{2 j+2}, \hbar \mu_{2 j+1}, 2 t\right) * M\left(\mu_{2 j+1}, \ell \mu_{2 j+2}, 2 t\right)}-1\right) \\
& +c\left(\frac{M_{r}\left(\mu_{2 j+2}, \ell \mu_{2 j+2}, t\right) * M_{r}\left(\mu_{2 j+1}, \hbar \mu_{2 j+1}, t\right)}{M_{r}\left(\mu_{2 j+1}, \mu_{2 j+2}, t\right) * M_{r}\left(\mu_{2 j+2}, \hbar \mu_{2 j+1}, 2 t\right) * M_{r}\left(\mu_{2 j+1}, \ell \mu_{2 j+2}, 2 t\right)}-1\right) \\
& +d\left(\frac{1}{M_{r}\left(\mu_{2 j+2}, \ell \mu_{2 j+2}, t\right)}-1+\frac{1}{M_{r}\left(\mu_{2 j+1}, \hbar \mu_{2 j+1}, t\right)}-1\right) \\
& =a\left(\frac{1}{M_{r}\left(\mu_{2 j+1}, \mu_{2 j+2}, t\right)}-1\right)+b\left(\frac{M_{r}\left(\mu_{2 j+1}, \mu_{2 j+2}, t\right)}{M_{r}\left(\mu_{2 j+2}, \mu_{2 j+2}, 2 t\right) * M_{r}\left(x_{2 j+1}, x_{2 j+3}, 2 t\right)}-1\right) \\
& +c\left(\frac{M_{r}\left(\mu_{2 j+2}, \mu_{2 j+3}, t\right) * M_{r}\left(\mu_{2 j+1}, \mu_{2 j+2}, t\right)}{M_{r}\left(\mu_{2 j+1}, \mu_{2 j+2}, t\right) * M_{r}\left(\mu_{2 j+2}, \mu_{2 j+2}, 2 t\right) * M_{r}\left(\mu_{2 j+1}, \mu_{2 j+3}, 2 t\right)}-1\right) \\
& +d\left(\frac{1}{M_{r}\left(\mu_{2 j+2}, \mu_{2 j+3}, t\right)}-1+\frac{1}{M_{r}\left(\mu_{2 j+1}, \mu_{2 j+2}, t\right)}-1\right) \\
& =a\left(\frac{1}{M_{r}\left(\mu_{2 j+1}, \mu_{2 j+2}, t\right)}-1\right)+b\left(\frac{M_{r}\left(\mu_{2 j+1}, \mu_{2 j+2}, t\right)}{M_{r}\left(\mu_{2 j+1}, \mu_{2 j+3}, 2 t\right)}-1\right) \\
& +c\left(\frac{M_{r}\left(\mu_{2 j+2}, \mu_{2 j+3}, t\right)}{M_{r}\left(\mu_{2 j+1}, \mu_{2 j+3}, 2 t\right)}-1\right)+d\left(\frac{1}{M_{r}\left(\mu_{2 j+2}, \mu_{2 j+3}, t\right)}-1+\frac{1}{M_{r}\left(\mu_{2 j+1}, \mu_{2 j+2}, t\right)}-1\right)
\end{aligned}
$$

Again, by Definition 2 (3), $M_{r}\left(\mu_{2 j+1}, \mu_{2 j+3}, 2 t\right) \geq M_{r}($ $\left.\mu_{2 j+1}, \mu_{2 j+2}, t\right) * M_{r}\left(\mu_{2 j+2}, \mu_{2 j+3}, t\right)$, for $t \gg \theta$. We have 


$$
\begin{aligned}
& \frac{1}{M_{r}\left(\mu_{2 j+2}, \mu_{2 j+3}, t\right)}-1 \\
& \leq a\left(\frac{1}{M_{r}\left(\mu_{2 j+1}, \mu_{2 j+2}, t\right)}-1\right) \\
& \quad+b\left(\frac{M_{r}\left(\mu_{2 j+1}, \mu_{2 j+2}, t\right)}{M_{r}\left(\mu_{2 j+1}, \mu_{2 j+2}, t\right) * M_{r}\left(\mu_{2 j+2}, \mu_{2 j+3}, t\right)}-1\right) \\
& \quad+c\left(\frac{M_{r}\left(\mu_{2 j+2}, \mu_{2 j+3}, t\right)}{M_{r}\left(\mu_{2 j+1}, \mu_{2 j+2}, t\right) * M_{r}\left(\mu_{2 j+2}, \mu_{2 j+3}, 2 t\right)}-1\right) \\
& \quad+d\left(\frac{1}{M_{r}\left(\mu_{2 j+1}, \mu_{2 j+2}, t\right)}-1+\frac{1}{M_{r}\left(\mu_{2 j+2}, \mu_{2 j+3}, t\right)}-1\right) .
\end{aligned}
$$

After simplification, we have

$\frac{1}{M_{r}\left(\mu_{2 j+2}, \mu_{2 j+3}, t\right)}-1 \leq \gamma\left(\frac{1}{M_{r}\left(\mu_{2 j+1}, \mu_{2 j+2}, t\right)}-1\right), \quad$ for $t \gg \theta$,

where the value of $\gamma$ is the same as in (9). Now, from (9) and (12) and by induction, we have

$$
\begin{aligned}
& \frac{1}{M_{r}\left(\mu_{2 j+2}, \mu_{2 j+3}, t\right)}-1 \\
& \quad \leq \gamma\left(\frac{1}{M_{r}\left(\mu_{2 j+1}, \mu_{2 j+2}, t\right)}-1\right) \\
& \quad \leq \gamma^{2}\left(\frac{1}{M_{r}\left(\mu_{2 j+1}, \mu_{2 j+2}, t\right)}-1\right) \leq \cdots \\
& \quad \leq \gamma^{2 j+2}\left(\frac{1}{M_{r}\left(\mu_{0}, \mu_{1}, t\right)}-1\right) \longrightarrow 0, \quad \text { as } j \longrightarrow \infty
\end{aligned}
$$

which yields that $\left(\mu_{j}\right)$ is a Cauchy sequence in $U$. Since $\left(U, M_{r}, *\right)$ is complete, there is $v_{1} \in U$ such that

$$
\lim _{j \longrightarrow \infty} M_{r}\left(\mu_{2 j+1}, v_{1}, t\right)=1, \quad \text { for } t \gg \theta
$$

Now, we prove that $\hbar v_{1}=v_{1}$. Since $M_{r}$ is triangular,

$$
\begin{aligned}
\frac{1}{M_{r}\left(v_{1}, \hbar v_{1}, t\right)}-1 \leq & \left(\frac{1}{M_{r}\left(v_{1}, \mu_{2 j+1}, t\right)}-1\right) \\
& +\left(\frac{1}{M_{r}\left(\mu_{2 j+1}, \hbar v_{1}, t\right)}-1\right), \text { for } t \gg \theta
\end{aligned}
$$

By (5), (14), and (16), for $t \gg \theta$,

$$
\begin{aligned}
\frac{1}{M_{r}\left(\mu_{2 j+1}, \hbar v_{1}, t\right)}-1= & \frac{1}{M_{r}\left(\ell \mu_{2 j}, \hbar v_{1}, t\right)}-1 \leq a\left(\frac{1}{M_{r}\left(\mu_{2 j}, v_{1}, t\right)}-1\right)+b\left(\frac{M_{r}\left(\mu_{2 j}, v_{1}, t\right)}{M_{r}\left(\mu_{2 j}, \hbar v_{1}, 2 t\right) * M_{r}\left(v_{1}, \ell \mu_{2 j}, 2 t\right)}-1\right) \\
& +c\left(\frac{1}{M_{r}\left(\mu_{2 j}, v, t\right) * M_{r}\left(\mu_{2 j}, \hbar v_{1}, 2 t\right) * M_{r}\left(v_{1}, \ell \mu_{2 j}, 2 t\right)}-1\right)+d\left(\frac{1}{M_{r}\left(\mu_{2 j}, \ell \mu_{2 j}, t\right)}-1+\frac{1}{M_{r}\left(v_{1}, \hbar v_{1}, t\right)}-1\right) \\
= & a\left(\frac{1}{M_{r}\left(\mu_{2 j}, v_{1}, t\right)}-1\right)+b\left(\frac{M_{r}\left(\mu_{2 j}, v_{1}, t\right)}{M_{r}\left(\mu_{2 j}, \hbar v_{1}, 2 t\right) * M_{r}\left(v_{1}, \mu_{2 j+1}, 2 t\right)}-1\right) \\
& +c\left(\frac{M_{r}\left(\mu_{2 j}, \mu_{2 j+1}, t\right) * M_{r}\left(v_{1}, \hbar v_{1}, t\right)}{M_{r}\left(\mu_{2 j}, v_{1}, t\right) * M_{r}\left(\mu_{2 j}, \hbar v_{1}, 2 t\right) * M_{r}\left(v_{1}, \mu_{2 j+1}, 2 t\right)}-1\right)+d\left(\frac{1}{M_{r}\left(\mu_{2 j}, \mu_{2 j+1}, t\right)}-1+\frac{1}{M_{r}\left(v_{1}, \hbar v_{1}, t\right)}-1\right) .
\end{aligned}
$$


Again, by Definition 2 (3), $M_{r}\left(\mu_{2 j}, \hbar v_{1}, 2 t\right) \geq M_{r}\left(\mu_{2 j}, v_{1}\right.$, $t) * M_{r}\left(v_{1}, \hbar v_{1}, t\right)$, for $t \gg \theta$. It follows that

$$
\begin{aligned}
\frac{1}{M_{r}\left(\mu_{2 j+1}, \hbar v_{1}, t\right)}-1 \leq a( & \left.\frac{1}{M_{r}\left(\mu_{2 j}, v_{1}, t\right)}-1\right)+b\left(\frac{M_{r}\left(\mu_{2 j}, v_{1}, t\right)}{M_{r}\left(\mu_{2 j}, v_{1}, t\right) * M_{r}\left(v_{1}, \hbar v_{1}, t\right) * M_{r}\left(v_{1}, \mu_{2 j+1}, 2 t\right)}-1\right) \\
& +c\left(\frac{M_{r}\left(\mu_{2 j}, \mu_{2 j+1}, t\right) * M_{r}\left(v_{1}, \hbar v_{1}, t\right)}{M_{r}\left(\mu_{2 j}, v_{1}, t\right) * M_{r}\left(\mu_{2 j}, v_{1}, t\right) * M_{r}\left(v_{1}, \hbar v_{1}, t\right) * M_{r}\left(v_{1}, \mu_{2 j+1}, 2 t\right)}-1\right) \\
& +d\left(\frac{1}{M_{r}\left(\mu_{2 j}, \mu_{2 j+1}, t\right)}-1+\frac{1}{M_{r}\left(v_{1}, \hbar v_{1}, t\right)}-1\right) \longrightarrow(b+d)\left(\frac{1}{M_{r}\left(v_{1}, \hbar v_{1}, t\right)}-1\right), \text { as } j \longrightarrow \infty .
\end{aligned}
$$

Then,

$$
\begin{aligned}
& \limsup _{j \rightarrow \infty}\left(\frac{1}{M_{r}\left(\mu_{2 j+1}, \hbar v_{1}, t\right)}-1\right) \\
& \quad \leq(b+d)\left(\frac{1}{M_{r}\left(v_{1}, \hbar v_{1}, t\right)}-1\right), \quad \text { for } t \gg \theta .
\end{aligned}
$$

This together with (17) and (16) implies

$\frac{1}{M_{r}\left(v_{1}, \hbar v_{1}, t\right)}-1 \leq(b+d)\left(\frac{1}{M_{r}\left(v_{1}, \hbar v_{1}, t\right)}-1\right), \quad$ for $t \gg \theta$.
Note that $(b+d)<1$ because $a+b+c+2 d<1$. Then, $M_{r}\left(v_{1}, \hbar v_{1}, t\right)=1$, that is, $\hbar v_{1}=v_{1}$. Similarly, we can show that $\ell v_{1}=v_{1}$ because $M_{r}$ is triangular. Therefore,

$$
\begin{aligned}
\frac{1}{M_{r}\left(v_{1}, \ell v_{1}, t\right)}-1 \leq & \left(\frac{1}{M_{r}\left(v_{1}, \mu_{2 j+2}, t\right)}-1\right) \\
& +\left(\frac{1}{M_{r}\left(\mu_{2 j+2}, \ell v_{1}, t\right)}-1\right), \text { for } t \gg \theta
\end{aligned}
$$

Now, again by (5), (14), and (16), one writes for $t \gg \theta$

$$
\begin{aligned}
\frac{1}{M_{r}\left(\mu_{2 j+2}, \ell v_{1}, t\right)}-1= & \frac{1}{M_{r}\left(\ell v_{1}, \hbar \mu_{2 j+1}, t\right)}-1 \leq a\left(\frac{1}{M_{r}\left(v_{1}, \mu_{2 j+1}, t\right)}-1\right)+b\left(\frac{M_{r}\left(v_{1}, \mu_{2 j+1}, t\right)}{M_{r}\left(v_{1}, \hbar \mu_{2 j+1}, 2 t\right) * M_{r}\left(\mu_{2 j+1}, \ell v_{1}, 2 t\right)}-1\right) \\
& +c\left(\frac{M_{r}\left(v_{1}, \ell v_{1}, t\right) * M_{r}\left(\mu_{2 j+1}, \hbar \mu_{2 j+1}, t\right)}{M_{r}\left(v_{1}, \mu_{2 j+1}, t\right) * M_{r}\left(v_{1}, \hbar \mu_{2 j+1}, 2 t\right) * M_{r}\left(\mu_{2 j+1}, \ell v_{1}, 2 t\right)}-1\right)+d\left(\frac{1}{M_{r}\left(v_{1}, \ell v_{1}, t\right)}-1+\frac{1}{M_{r}\left(\mu_{2 j+1}, \hbar \mu_{2 j+1}, t\right)}-1\right) \\
= & a\left(\frac{1}{M_{r}\left(v_{1}, \mu_{2 j+1}, t\right)}-1\right)+b\left(\frac{M_{r}\left(v_{1}, \mu_{2 j+1}, t\right)}{M_{r}\left(v_{1}, \mu_{2 j+2}, 2 t\right) * M_{r}\left(\mu_{2 j+1}, \ell v_{1}, 2 t\right)}-1\right) \\
& +c\left(\frac{M_{r}\left(v_{1}, \ell v_{1}, t\right) * M_{r}\left(\mu_{2 j+1}, \mu_{2 j+2}, t\right)}{M_{r}\left(v_{1}, \mu_{2 j+1}, t\right) * M_{r}\left(v_{1}, \mu_{2 j+2}, 2 t\right) * M_{r}\left(\mu_{2 j+1}, \ell v_{1}, 2 t\right)}-1\right)+d\left(\frac{1}{M_{r}\left(v_{1}, \ell v_{1}, t\right)}-1+\frac{1}{M_{r}\left(\mu_{2 j+1}, \mu_{2 j+2}, t\right)}-1\right) .
\end{aligned}
$$


Again, by Definition 2 (3), $M_{r}\left(\mu_{2 j+1}, \ell v_{1}, 2 t\right) \geq M_{r}\left(\mu_{2 j+1}\right.$ $\left., v_{1}, t\right) * M_{r}\left(v_{1}, \ell v_{1}, t\right)$, for $t \gg \theta$. It follows that

$$
\begin{aligned}
\frac{1}{M_{r}\left(\mu_{2 j+1}, \ell v_{1}, t\right)}-1 \leq & a\left(\frac{1}{M_{r}\left(v_{1}, \mu_{2 j+1}, t\right)}-1\right)+b\left(\frac{M_{r}\left(v_{1}, \mu_{2 j+1}, t\right)}{M_{r}\left(v_{1}, \mu_{2 j+2}, 2 t\right) * M_{r}\left(\mu_{2 j+1}, v_{1}, t\right) * M_{r}\left(v_{1}, \ell v_{1}, t\right)}-1\right) \\
& +c\left(\frac{M_{r}\left(v_{1}, \ell v_{1}, t\right) * M_{r}\left(\mu_{2 j+1}, \mu_{2 j+2}, t\right)}{M_{r}\left(v_{1}, \mu_{2 j+1}, t\right) * M_{r}\left(v_{1}, \mu_{2 j+2}, 2 t\right) * M_{r}\left(\mu_{2 j+1}, v_{1}, t\right) * M_{r}\left(v_{1}, \ell v_{1}, t\right)}-1\right) \\
& +d\left(\frac{1}{M_{r}\left(v_{1}, \ell v_{1}, t\right)}-1+\frac{1}{M_{r}\left(\mu_{2 j+1}, \mu_{2 j+2}, t\right)}-1\right) \longrightarrow(b+d)\left(\frac{1}{M_{r}\left(v_{1}, \ell v_{1}, t\right)}-1\right), \text { as } j \longrightarrow \infty .
\end{aligned}
$$

Then,

$$
\begin{aligned}
& \underset{j \longrightarrow \infty}{\limsup }\left(\frac{1}{M_{r}\left(\mu_{2 j+2}, \ell v_{1}, t\right)}-1\right) \\
& \quad \leq(b+d)\left(\frac{1}{M_{r}\left(v_{1}, \ell v_{1}, t\right)}-1\right), \text { for } t \gg \theta .
\end{aligned}
$$

This together with (22) and (16) implies

$\frac{1}{M_{r}\left(v_{1}, \ell v_{1}, t\right)}-1 \leq(b+d)\left(\frac{1}{M_{r}\left(v_{1}, \ell v_{1}, t\right)}-1\right), \quad$ for $t \gg \theta$.

Note that $(b+d)<1$ since $a+b+c+2 d<1$. Then, $M_{r}($ $\left.v_{1}, \ell v_{1}, t\right)=1$, that is, $\ell v_{1}=v_{1}$.

Hence, $v_{1}$ is a common fixed point of $\ell$ and $\hbar$.

Example 1. Let $U=[0, \infty), *$ be a continuous $t$-norm and $M_{r}: U^{2} \times(0, \infty) \longrightarrow[0,1]$ be written as

$$
M_{r}(\mu, v, t)=\frac{t}{t+|\mu-v|}, \quad \forall \mu, v \in U, t \gg \theta .
$$

Then, easily one can verify that $M_{r}$ is triangular and ( $U$ $\left., M_{r}, *\right)$ is a complete FCM space. Now, we define $\ell, \hbar: U$ $\longrightarrow U$ by

$$
\ell(\mu)=\hbar(\mu)= \begin{cases}\frac{3 \mu}{8}, & \text { if } \mu \in[0,1), \\ \frac{4 \mu}{5}+\frac{7}{5}, & \text { if } \mu \in[1, \infty) .\end{cases}
$$

Then, for $t \gg \theta$, we have

$$
\frac{1}{M_{r}(\ell(\mu), \hbar(\nu), t)}-1=\left|\frac{\ell(\mu)-\hbar(v)}{t}\right|=\frac{3}{8}\left(\frac{1}{M_{r}(\mu, v, t)}-1\right) .
$$

Hence, the pair of self-mapping $(\ell, \hbar)$ is a fuzzy conecontraction. Now, from Definition 2 (3), $M_{r}(\mu, \hbar v, 2 t) \geq M_{r}$ $(\mu, v, t) * M_{r}(\nu, \hbar \nu, t)$ and $M_{r}(\nu, \ell \mu, 2 t) \geq M_{r}(\nu, \mu, t) * M_{r}($ $\mu, \ell \mu, t)$, for $t \gg \theta$. We get the following:

$$
\begin{aligned}
& \left(\frac{M_{r}(\mu, v, t)}{M_{r}(\mu, \hbar v, 2 t) * M_{r}(\nu, \ell \mu, 2 t)}-1\right) \\
& \quad \leq \frac{1}{M_{r}(\mu, v, t) * M_{r}(\mu, \ell \mu, t) * M_{r}(v, \hbar v, t)}-1 \\
& \quad=\frac{1}{(t /(t+|\mu-v|))(t /(t+|\mu-\ell \mu|))(t /(t+|v-\hbar v|))}-1 \\
& \quad=\frac{(t+|\mu-v|)(t+|\mu-\ell \mu|)(t+|v-\hbar v|)}{t^{3}}-1 \\
& \quad=\frac{(t+|\mu-v|)[(5 t / 8)(\mu+v)+(25 / 64) \mu v]+t^{2}|\mu-v|}{t^{3}} \\
& \quad=\frac{5(t+|\mu-v|)[8 t(\mu+v)+5 \mu v]}{64 t^{3}}+\frac{|\mu-v|}{t},
\end{aligned}
$$

$$
\begin{aligned}
& \left(\frac{M_{r}(\mu, \ell \mu, t) * M_{r}(\nu, \hbar v, t)}{M_{r}(\mu, v, t) * M_{r}(\mu, \hbar v, 2 t) * M_{r}(\nu, \ell \mu, 2 t)}-1\right) \\
& \quad \leq \frac{1}{\left(M_{r}(\mu, \nu, t)\right)^{3}}-1=\frac{1}{(t /(t+|\mu-v|))^{3}}-1 \\
& \quad=\frac{(t+|\mu-v|)^{3}}{t^{3}}-1=\frac{(|\mu-v|)^{3}+3 t|\mu-v|(t+|\mu-v|)}{t^{3}},
\end{aligned}
$$




$$
\begin{aligned}
& \left(\frac{1}{M_{r}(\mu, \ell \mu, t)}-1+\frac{1}{M_{r}(\nu, \hbar v, t)}-1\right) \\
& \quad=\frac{1}{t /(t+|\mu-\ell \mu|)}-1+\frac{1}{t /(t+|v-\hbar v|)}-1=\frac{5(\mu+v)}{8 t} .
\end{aligned}
$$

Hence, from the above, we conclude that all the conditions of Theorem 7 are satisfied with $a=3 / 8, b=c=1 / 6$, and $d=1 / 8$. The mappings $\ell$ and $\hbar$ have a common fixed point, i.e., $\ell(7)=\hbar(7)=7 \in[0, \infty)$.

Putting $b=0$ in Theorem 7 , we get the following corollary.

Corollary 8. Let $\left(U, M_{r}, *\right)$ be a complete FCM space in which $M_{r}$ is triangular. Let $\ell, \hbar: U \longrightarrow U$ be a pair of self-mappings so that

$$
\begin{aligned}
& \frac{1}{M_{r}(\ell \mu, \hbar \nu, t)}-1 \\
& \leq a\left(\frac{1}{M_{r}(\mu, v, t)}-1\right) \\
& \quad+c\left(\frac{M_{r}(\mu, \ell \mu, t) * M_{r}(\nu, \hbar \nu, t)}{M_{r}(\mu, v, t) * M_{r}(\mu, \hbar \nu, 2 t) * M_{r}(\nu, \ell \mu, 2 t)}-1\right) \\
& \quad+d\left(\frac{1}{M_{r}(\mu, \ell \mu, t)}-1+\frac{1}{M_{r}(\nu, \hbar v, t)}-1\right),
\end{aligned}
$$

for all $\mu, v \in U, t \gg \theta, a \in(0,1)$, and $c, d \geq 0$ with $(a+c+2 d$ )$<1$. Then, $\ell$ and $\hbar$ have a common fixed point in $U$.

In the following corollary, we prove that the mappings $\ell$ and $\hbar$ have a unique common fixed point in $U$ by using the constant $c=0$ in Theorem 7 .

Corollary 9. Let $\left(U, M_{r}, *\right)$ be a complete FCM space in which $M_{r}$ is triangular. Let $\ell, \hbar: U \longrightarrow U$ be a pair of self-mappings so that

$$
\begin{aligned}
\frac{1}{M_{r}(\ell \mu, \hbar v, t)}-1 \leq & a\left(\frac{1}{M_{r}(\mu, v, t)}-1\right) \\
& +b\left(\frac{M_{r}(\mu, v, t)}{M_{r}(\mu, \hbar v, 2 t) * M_{r}(\nu, \ell \mu, 2 t)}-1\right) \\
& +d\left(\frac{1}{M_{r}(\mu, \ell \mu, t)}-1+\frac{1}{M_{r}(v, \hbar v, t)}-1\right),
\end{aligned}
$$

for all $\mu, v \in U, t \gg \theta, a \in(0,1)$, and $b, d \geq 0$ with $a+b+2 d$ $<1$. Hence, $\ell$ and $\hbar$ have a unique common fixed point in $U$.

Proof. It follows from the proof of Theorem 7 that $v_{1}$ is a common fixed point of $\ell$ and $\hbar$ in $U$. For uniqueness, let $u_{1}$ be another common fixed point of $\ell$ and $\hbar$ in $U$ such that $\ell$ $u_{1}=\hbar u_{1}=u_{1}$ and $\ell v_{1}=\hbar v_{1}=v_{1}$. Then, by view of (32),

$$
\begin{aligned}
\frac{1}{M_{r}\left(u_{1}, v_{1}, t\right)}-1= & \frac{1}{M_{r}\left(\ell u_{1}, \hbar v_{1}, t\right)}-1 \\
\leq & a\left(\frac{1}{M_{r}\left(u_{1}, v_{1}, t\right)}-1\right) \\
& +b\left(\frac{M_{r}\left(u_{1}, v_{1}, t\right)}{M_{r}\left(u_{1}, \hbar v_{1}, 2 t\right) * M_{r}\left(v_{1}, \ell u_{1}, 2 t\right)}-1\right) \\
& +d\left(\frac{1}{M_{r}\left(\mu_{1}, \ell \mu_{1}, t\right)}-1+\frac{1}{M_{r}\left(v_{1}, \hbar v_{1}, t\right)}-1\right) .
\end{aligned}
$$

By Definition 2 (3),

$$
\begin{aligned}
& M_{r}\left(v_{1}, \ell u_{1}, 2 t\right) \geq M_{r}\left(v_{1}, u_{1}, t\right) * M_{r}\left(u_{1}, \ell u_{1}, t\right) \\
& =M_{r}\left(v_{1}, u_{1}, t\right) * 1=M_{r}\left(v_{1}, u_{1}, t\right), \quad \text { for } t \gg \theta, \\
& M_{r}\left(u_{1}, \hbar v_{1}, 2 t\right) \geq M_{r}\left(u_{1}, v_{1}, t\right) * M_{r}\left(v_{1}, \hbar v_{1}, t\right) \\
& =M_{r}\left(u_{1}, v_{1}, t\right) * 1=M_{r}\left(u_{1}, v_{1}, t\right), \quad \text { for } t \gg \theta \text {. }
\end{aligned}
$$

It follows that

$$
\begin{aligned}
\frac{1}{M_{r}\left(u_{1}, v_{1}, t\right)}-1 \leq & a\left(\frac{1}{M_{r}\left(u_{1}, v_{1}, t\right)}-1\right) \\
& +b\left(\frac{M_{r}\left(u_{1}, v_{1}, t\right)}{M_{r}\left(u_{1}, v_{1}, t\right) * M_{r}\left(v_{1}, u_{1}, t\right)}-1\right) \\
& +d\left(\frac{1}{M_{r}\left(\mu_{1}, \mu_{1}, t\right)}-1+\frac{1}{M_{r}\left(v_{1}, v_{1}, t\right)}-1\right) \\
= & (a+b)\left(\frac{1}{M_{r}\left(u_{1}, v_{1}, t\right)}-1\right) \\
= & (a+b)\left(\frac{1}{M_{r}\left(\ell u_{1}, \hbar v_{1}, t\right)}-1\right) \\
\leq & (a+b)^{2}\left(\frac{1}{M_{r}\left(u_{1}, v_{1}, t\right)}-1\right) \leq \cdots \\
\leq & (a+b)^{j}\left(\frac{1}{M_{r}\left(u_{1}, v_{1}, t\right)}-1\right) \longrightarrow 0, \quad \text { as } j \longrightarrow \infty .
\end{aligned}
$$

Since $a+b<1$, one writes $M_{r}\left(u_{1}, v_{1}, t\right)=1$, i.e., $u_{1}=v_{1}$ for $t \gg \theta$.

Corollary 10. Let $\left(U, M_{r}, *\right)$ be a complete FCM space in which $M_{r}$ is triangular. Let $\ell, \hbar: U \longrightarrow U$ be a pair of selfmappings so that

$$
\begin{aligned}
\frac{1}{M_{r}(\ell \mu, \hbar v, t)}-1 \leq & a\left(\frac{1}{M_{r}(\mu, v, t)}-1\right) \\
& +d\left(\frac{1}{M_{r}(\mu, \ell \mu, t)}-1+\frac{1}{M_{r}(\nu, \hbar \nu, t)}-1\right)
\end{aligned}
$$

for all $\mu, v \in U, t \gg \theta, a \in(0,1)$, and $d \geq 0$ with $a+2 d<1$. Then, $\ell$ and $\hbar$ have a unique common fixed point in $U$. 
Example 2. As in Example 1, let $M_{r}: U^{2} \times(0, \infty) \longrightarrow[0,1]$ be defined by

$$
M_{r}(\mu, v, t)=\frac{t}{t+|(\mu-v) / 2|}, \quad \forall \mu, v \in U, t \gg \theta
$$

Then, easily one can verify that $M_{r}$ is triangular and ( $U$ $\left., M_{r}, *\right)$ is a complete FCM space. Now, we define selfmappings $\ell, \hbar: U \longrightarrow U$ by

$$
\begin{aligned}
& l(\mu)= \begin{cases}\frac{2 \mu}{3}+\frac{1}{3}, & \mu \in[0,1], \\
\frac{4 \mu}{5}+\frac{8}{5}, & \mu \in(1, \infty),\end{cases} \\
& \hbar(v)= \begin{cases}\frac{2 v}{3}+\frac{1}{3}, & v \in[0,1], \\
\frac{5 v}{6}+\frac{4}{3}, & v \in(1, \infty) .\end{cases}
\end{aligned}
$$

Then, from (36), for $t \gg \theta$, we have

$$
\begin{aligned}
\left(\frac{1}{M_{r}(\ell \mu, \hbar v, t)}-1\right)= & \left|\frac{(\mu-v)}{3 t}\right| \leq \frac{2}{3}\left|\frac{(\mu-v)}{2 t}\right|+\left|\frac{(\mu+v-2)}{42 t}\right| \\
\leq & \frac{2}{3}\left|\frac{(\mu-v)}{2 t}\right|+\frac{1}{7}\left|\frac{(\mu+v-2)}{6 t}\right| \\
= & a\left(\frac{1}{M_{r}(\mu, v, t)}-1\right) \\
& +d\left(\frac{1}{M_{r}(\mu, \ell \mu, t)}-1+\frac{1}{M_{r}(\nu, \hbar v, t)}-1\right) .
\end{aligned}
$$

Hence, all the conditions of Corollary 10 are satisfied with $a=2 / 3$ and $d=1 / 7$. The mappings $\ell$ and $\hbar$ have a common fixed point, i.e., $\ell(8)=\hbar(8)=8 \in[0, \infty)$.

Theorem 11. Let $\left(U, M_{r}, *\right)$ be a complete FCM space in which $M_{r}$ is triangular. Let $\ell, \hbar: U \longrightarrow U$ be a pair of selfmappings so that

$$
\begin{aligned}
& \frac{1}{M_{r}(\ell \mu, \hbar v, t)}-1 \\
& \leq a\left(\frac{1}{M_{r}(\mu, v, t)}-1\right) \\
& \quad+b\left(\frac{M_{r}(\mu, v, t)}{M_{r}(\mu, \hbar v, 2 t) * M_{r}(\nu, \ell \mu, 2 t)}-1\right) \\
& \quad+c\left(\frac{M_{r}(\mu, \ell \mu, t) * M_{r}(\nu, \hbar v, t)}{M_{r}(\mu, v, t) * M_{r}(\mu, \hbar v, 2 t) * M_{r}(\nu, \ell \mu, 2 t)}-1\right) \\
& \quad+d\left(\frac{1}{M_{r}(\nu, \ell \mu, t)}-1+\frac{1}{M_{r}(\mu, \hbar v, t)}-1\right),
\end{aligned}
$$

for all $\mu, v \in U, t \gg \theta, a \in(0,1)$, and $b, c, d \geq 0$ with $a+$ $b+c+2 d<1$. Then, $\ell$ and $\hbar$ have a common fixed point in $U$.
Proof. The proof is similar as the proof of Theorem 7.

Corollary 12. Let $\left(U, M_{r}, *\right)$ be a complete FCM space in which $M_{r}$ is triangular. Let $\ell, \hbar: U \longrightarrow U$ be a pair of selfmappings so that

$$
\begin{aligned}
& \frac{1}{M_{r}(\ell \mu, \hbar v, t)}-1 \\
& \leq a\left(\frac{1}{M_{r}(\mu, v, t)}-1\right) \\
& \quad+c\left(\frac{M_{r}(\mu, \ell \mu, t) * M_{r}(\nu, \hbar v, t)}{M_{r}(\mu, v, t) * M_{r}(\mu, \hbar v, 2 t) * M_{r}(\nu, \ell \mu, 2 t)}-1\right) \\
& \quad+d\left(\frac{1}{M_{r}(\nu, \ell \mu, t)}-1+\frac{1}{M_{r}(\mu, \hbar v, t)}-1\right),
\end{aligned}
$$

for all $\mu, v \in U, t \gg \theta, a \in(0,1)$, and $c, d \geq 0$ with $(a+c$ $+2 d)<1$. Then, $\ell$ and $\hbar$ have a common fixed point in $U$.

Corollary 13. Let $\left(U, M_{r}, *\right)$ be a complete FCM space in which $M_{r}$ is triangular. Let $\ell, \hbar: U \longrightarrow U$ be a pair of selfmappings so that

$$
\begin{aligned}
\frac{1}{M_{r}(\ell \mu, \hbar v, t)}-1 \leq & a\left(\frac{1}{M_{r}(\mu, v, t)}-1\right) \\
& +b\left(\frac{M_{r}(\mu, v, t)}{M_{r}(\mu, \hbar \nu, 2 t) * M_{r}(\nu, \ell \mu, 2 t)}-1\right) \\
& +d\left(\frac{1}{M_{r}(\nu, \ell \mu, t)}-1+\frac{1}{M_{r}(\mu, \hbar v, t)}-1\right)
\end{aligned}
$$

$\forall \mu, v \in U, t \gg \theta, a \in(0,1)$, and $b, d \geq 0$ with $(a+b+2 d)$ $<1$. Then, $\ell$ and $\hbar$ have a unique common fixed point in $U$.

Proof. It is as the proof of Theorem 7. Let $v_{1}$ be a common fixed point of $\ell$ and $\hbar$ in $U$. Let $u_{1}$ be another common fixed point of $\ell$ and $\hbar$ in $U$ such that $\ell u_{1}=\hbar u_{1}=u_{1}$ and $\ell v_{1}=\hbar v_{1}$ $=v_{1}$. Then, by view of (42),

$$
\begin{aligned}
\frac{1}{M_{r}\left(u_{1}, v_{1}, t\right)}-1= & \frac{1}{M_{r}\left(\ell u_{1}, \hbar v_{1}, t\right)}-1 \\
\leq & a\left(\frac{1}{M_{r}\left(u_{1}, v_{1}, t\right)}-1\right) \\
& +b\left(\frac{M_{r}\left(u_{1}, v_{1}, t\right)}{M_{r}\left(u_{1}, \hbar v_{1}, 2 t\right) * M_{r}\left(v_{1}, \ell u_{1}, 2 t\right)}-1\right) \\
& +d\left(\frac{1}{M_{r}\left(v_{1}, \ell u_{1}, t\right)}-1+\frac{1}{M_{r}\left(u_{1}, \hbar v_{1}, t\right)}-1\right) .
\end{aligned}
$$

By Definition 2 (3),

$$
\begin{aligned}
M_{r}\left(v_{1}, \ell u_{1}, 2 t\right) & \geq M_{r}\left(v_{1}, u_{1}, t\right) * M_{r}\left(u_{1}, \ell u_{1}, t\right) \\
& =M_{r}\left(v_{1}, u_{1}, t\right) * 1=M_{r}\left(v_{1}, u_{1}, t\right), \quad \text { for } t \gg \theta,
\end{aligned}
$$




$$
\begin{aligned}
M_{r}\left(u_{1}, \hbar v_{1}, 2 t\right) & \geq M_{r}\left(u_{1}, v_{1}, t\right) * M_{r}\left(v_{1}, \hbar v_{1}, t\right) \\
& =M_{r}\left(u_{1}, v_{1}, t\right) * 1=M_{r}\left(u_{1}, v_{1}, t\right), \quad \text { for } t \gg \theta .
\end{aligned}
$$

It follows that

$$
\begin{aligned}
& \frac{1}{M_{r}\left(u_{1}, v_{1}, t\right)}-1 \\
& \leq a\left(\frac{1}{M_{r}\left(u_{1}, v_{1}, t\right)}-1\right) \\
& \quad+b\left(\frac{M_{r}\left(u_{1}, v_{1}, t\right)}{M_{r}\left(u_{1}, v_{1}, t\right) * M_{r}\left(v_{1}, u_{1}, t\right)}-1\right) \\
& \quad+d\left(\frac{1}{M_{r}\left(v_{1}, u_{1}, t\right)}-1+\frac{1}{M_{r}\left(u_{1}, v_{1}, t\right)}-1\right) \\
& =(a+b+2 d)\left(\frac{1}{M_{r}\left(u_{1}, v_{1}, t\right)}-1\right), \quad \text { for } t \gg \theta .
\end{aligned}
$$

Since $0<(a+b+2 d)<1, M_{r}\left(u_{1}, v_{1}, t\right)=1$, i.e., $u_{1}=v_{1}$.

Corollary 14. Let $\left(U, M_{r}, *\right)$ be a complete FCM space in which $M_{r}$ is triangular. Let $\ell, \hbar: U \longrightarrow U$ be a pair of selfmappings so that

$$
\begin{aligned}
\frac{1}{M_{r}(\ell \mu, \hbar \nu, t)}-1 \leq & a\left(\frac{1}{M_{r}(\mu, v, t)}-1\right) \\
& +d\left(\frac{1}{M_{r}(\nu, \ell \mu, t)}-1+\frac{1}{M_{r}(\mu, \hbar \nu, t)}-1\right),
\end{aligned}
$$

for all $\mu, v \in U, t \gg \theta, a \in(0,1)$, and $d \geq 0$ with $a+2 d<1$. Then, $\ell$ and $\hbar$ have a unique common fixed point in $U$.

Example 3. Let $U=[0,1]$. As in Example 2, we define selfmappings $\ell, \hbar: U \longrightarrow U$ by

$$
\begin{aligned}
& \ell(\mu)= \begin{cases}\frac{2 \mu}{5}+\frac{1}{7}, & \mu \in\left[0, \frac{1}{2}\right], \\
\frac{3 \mu}{4}+\frac{3}{16}, & \mu \in\left(\frac{1}{2}, 1\right],\end{cases} \\
& \hbar(v)= \begin{cases}\frac{2 v}{5}+\frac{1}{7}, & v \in\left[0, \frac{1}{2}\right], \\
\frac{2 v}{3}+\frac{1}{4}, & v \in\left(\frac{1}{2}, 1\right] .\end{cases}
\end{aligned}
$$

Now, from (46), for $t \gg \theta$, we have

$$
\begin{aligned}
\frac{1}{M_{r}(\ell \mu, \hbar v, t)}-1= & \left|\frac{\ell \mu-\hbar v}{2 t}\right|=\left|\frac{\mu-v}{5 t}\right| \leq \frac{2}{5}\left|\frac{\mu-v}{2 t}\right| \\
& +\frac{2}{7}\left|\frac{21(\mu+v)-10}{70 t}\right| \\
\leq & \frac{2}{5}\left|\frac{\mu-v}{2 t}\right|+\frac{2}{7}\left(\left|\frac{v-(2 \mu / 5)-(1 / 7)}{2 t}\right|\right. \\
& \left.+\left|\frac{\mu-(2 v / 5)-(1 / 7)}{2 t}\right|\right) \\
= & a\left(\frac{1}{M_{r}(\nu, \mu, t)}-1\right)+d\left(\frac{1}{M_{r}(\nu, \ell \mu, t)}-1\right. \\
& \left.+\frac{1}{M_{r}(\mu, \hbar v, t)}-1\right) .
\end{aligned}
$$

Hence, all the conditions of Corollary 14 are satisfied with $a=2 / 5$ and $d=2 / 7$. The mappings $\ell$ and $\hbar$ have a common fixed point, i.e., $\ell(3 / 4)=\hbar(3 / 4)=3 / 4 \in[0,1]$.

\section{Application}

In this section, we present an application on Fredholm integral equations. Let $U=C([0, \eta], \mathbb{R})$ be the space of all $\mathbb{R}$ -valued continuous functions on the interval $[0, \eta]$, where 0 $<\eta \in \mathbb{R}$. The Fredholm integral equations are

$$
\begin{gathered}
\mu(\tau)=\int_{0}^{\eta} K_{1}(\tau, v, \mu(v)) d v, \\
\nu(\tau)=\int_{0}^{\eta} K_{2}(\tau, v, v(v)) d v, \\
\forall \mu, v \in U,
\end{gathered}
$$

where $\tau \in[0, \eta]$ and $K_{1}, K_{2}:[0, \eta] \times[0, \eta] \times \mathbb{R} \longrightarrow \mathbb{R}$. The induced metric $d: U^{2} \longrightarrow \mathbb{R}$ be defined as

$d(\mu, v)=\sup _{\tau \in[0, \eta]}|\mu(\tau)-v(\tau)|=\|\mu-v\|, \quad$ where $\mu, v \in C([0, \eta], \mathbb{R})=U$.

The binary operation $*$ is defined by $\alpha * \lambda=\alpha \lambda$ for all $\alpha$ ,$\lambda \in[0, \eta]$. A standard fuzzy metric $M_{r}: U^{2} \times(0, \infty) \longrightarrow[0$, 1] is given as

$$
M_{r}(\mu, v, t)=\frac{t}{t+d(\mu, v)}, \quad \text { for } t>0, \forall \mu, v \in U
$$

Then, easily one can verify that $M_{r}$ is triangular and ( $U$ $\left., M_{r}, *\right)$ is a complete FCM space. 
Theorem 15. The two FIEs are

$$
\begin{aligned}
& \mu(\tau)=\int_{0}^{\eta} K_{1}(\tau, v, x(v)) d v, \\
& v(\tau)=\int_{0}^{\eta} K_{2}(\tau, v, v(v)) d v,
\end{aligned}
$$

where $\tau \in[0,1]$ and $\mu, v \in U$. Assume that $K_{1}, K_{2}:[0,1] \times$ $[0,1] \times \mathbb{R} \longrightarrow \mathbb{R}$ are such that $A_{\mu}, B_{v} \in \mathbf{E}$ for every $\mu, v \in \mathbf{E}$, where

$$
\begin{aligned}
& A_{\mu}(\tau)=\int_{0}^{\eta} K_{l}(\tau, v, \mu(v)) d v, \\
& B_{v}(\tau)=\int_{0}^{\eta} K_{2}(\tau, v, v(v)) d v .
\end{aligned}
$$

If there exists $\beta \in(0,1)$ such that for all $\mu, v \in U$,

$$
\left\|A_{\mu}-B_{v}\right\| \leq \beta N(\ell, \hbar, \mu, v),
$$

where

$$
N(\ell, \hbar, \mu, v)=\max \left\{\begin{array}{c}
\|\mu-v\|,\left\|\mu-A_{\mu}\right\|+\left\|v-B_{v}\right\|, \frac{1}{t^{2}}\left(3 t\|\mu-v\|^{2}+\|\mu-v\|^{3}\right), \\
\frac{1}{t^{2}}(t+\|\mu-v\|)\left(t\left\|\mu-A_{\mu}\right\|+t\left\|v-B_{v}\right\|+\left\|\mu-A_{\mu}\right\| \cdot\left\|v-B_{v}\right\|\right)
\end{array}\right\} .
$$

Then, the two FIEs defined in (49) have a common solution in $U$.

Proof. Define the mappings $\ell, \hbar: \mathbf{E} \longrightarrow \mathbf{E}$ by

$$
\begin{aligned}
& \ell(\mu)=A_{\mu}, \\
& \hbar(v)=B_{v} .
\end{aligned}
$$

The FIEs in (49) have a common solution if and only if $\ell$ and $\hbar$ have a common fixed point in $U$. Now, we have to show that Theorem 7 is applied to the integral operators $\ell$ and $\hbar$. Then, for all $\mu, v \in U$, we have the following four cases.

(a) If $N(\ell, \hbar, \mu, v)=\|\mu-v\|$ in (55), then from (51) and (54), we have

$$
\begin{aligned}
\frac{1}{M_{r}(\ell \mu, \hbar v, t)}-1 & =\frac{d(\ell \mu, \hbar v)}{t} \leq \beta \frac{N(\ell, \hbar, \mu, v)}{t} \\
& =\beta \frac{\|\mu-v\|}{t}=\beta\left(\frac{1}{M_{r}(\mu, v, t)}-1\right) .
\end{aligned}
$$

This implies that

$$
\frac{1}{M_{r}(\ell \mu, \hbar \nu, t)}-1 \leq \beta\left(\frac{1}{M_{r}(\mu, v, t)}-1\right), \quad \text { for } t \gg \theta
$$

for all $\mu, v \in U$ such that $\ell \mu \neq \hbar v$. It is obvious that the inequality (58) holds if $\ell \mu=\hbar \nu$. Thus, the integral operators $\ell$ and $\hbar$ satisfy all the conditions of Theorem 7 with $\beta=a$ and $b=c=d=0$ in (5). The integral operators $\ell$ and $\hbar$ have a common fixed point, i.e., (49) has a common solution in $U$.

(b) If $N(\ell, \hbar, \mu, v)=\left(1 / t^{2}\right)(t+\|\mu-v\|)\left(t\left\|\mu-A_{\mu}\right\|+t \| v\right.$ $\left.-B_{v}\|+\| \mu-A_{\mu}\|\cdot\| v-B_{v} \|\right)$ in (55), then from (51) and (54), we have

$$
\begin{aligned}
\frac{1}{M_{r}(\ell \mu, \hbar v, t)}-1= & \frac{d(\ell \mu, \hbar v)}{t} \\
\leq & \beta \frac{N(\ell, \hbar, \mu, v)}{t} \\
= & \beta \frac{1}{t^{3}}(t+\|\mu-v\|)\left(t\left\|\mu-A_{\mu}\right\|\right. \\
& \left.+t\left\|v-B_{v}\right\|+\left\|\mu-A_{\mu}\right\| \cdot\left\|v-B_{v}\right\|\right) .
\end{aligned}
$$

It yields that

$$
\begin{aligned}
\frac{1}{M_{r}(\ell \mu, \hbar v, t)}-1 \leq & \beta \frac{1}{t^{3}}(t+\|\mu-v\|)\left(t\left\|\mu-A_{\mu}\right\|\right. \\
& \left.+t\left\|v-B_{v}\right\|+\left\|\mu-A_{\mu}\right\| \cdot\left\|v-B_{v}\right\|\right),
\end{aligned}
$$


for all $\mu, v \in U$ and for $t \gg \theta$. Here, we simplify the term $\left(\left(M_{r}(\mu, \nu, t)\right) /\left(M_{r}(\mu, \hbar \nu, 2 t) * M_{r}(\nu, \ell \mu, 2 t)\right)\right)-1$ by using Definition 2 (3) and (51), for $t \gg \theta$, we have

$$
\begin{aligned}
\frac{M_{r}(\mu, v, t)}{M_{r}(\mu, \hbar v, 2 t) * M_{r}(v, \ell \mu, 2 t)}-1 \leq & \frac{M_{r}(\mu, v, t)}{M_{r}(\mu, v, t) * M_{r}(v, \hbar v, t) * M_{r}(\nu, \mu, t) * M_{r}(\mu, \ell \mu, t)}-1 \\
= & \frac{1}{M_{r}(\mu, v, t) * M_{r}(v, \hbar v, t) * M_{r}(\mu, \ell \mu, t)}-1 \\
& =\frac{(t+\|\mu-v\|)(t+\|v-\hbar v\|)(t+\|\mu-\ell \mu\|)}{t^{3}}-1 \\
& =\frac{1}{t^{3}}\left(\begin{array}{c}
t^{2}\|\mu-v\|+t^{2}(\|v-\hbar v\|+\|\mu-\ell \mu\|)+t\|\mu-v\|(\|v-\hbar v\|+\|\mu-\ell \mu\|) \\
t\|\mu-\ell \mu\| \cdot\|v-\hbar v\|+\|\mu-v\| \cdot\|\mu-\ell \mu\| \cdot\|v-\hbar v\|
\end{array}\right) \\
& =\frac{1}{t^{3}}\left(t^{2}\|\mu-v\|+(t+\|\mu-v\|)(t\|v-\hbar v\|+t\|\mu-\ell \mu\|+\|\mu-\ell \mu\| \cdot\|v-\hbar v\|)\right) .
\end{aligned}
$$

This implies that

$$
\begin{aligned}
& \frac{M_{r}(\mu, v, t)}{M_{r}(\mu, \hbar v, 2 t) * M_{r}(\nu, \ell \mu, 2 t)}-1 \\
& \leq \frac{1}{t^{3}}\left(t^{2}\|\mu-v\|+(t+\|\mu-v\|)\right. \\
& \quad \cdot(t\|v-\hbar v\|+t\|\mu-\ell \mu\|+\|\mu-\ell \mu\| \cdot\|v-\hbar v\|)),
\end{aligned}
$$
have

for all $\mu, v \in U$ and for $t \gg \theta$. Now, from (60) and (62), we $\frac{1}{M_{r}(\ell \mu, \hbar v, t)}-1 \leq \beta\left(\frac{M_{r}(\mu, v, t)}{M_{r}(\mu, \ell \mu, t) * M_{r}(v, \hbar v, 2 t)}-1\right), \quad$ for $t \gg \theta$,

for all $\mu, v \in U$ such that $\ell \mu \neq \hbar \nu$. It is obvious that the inequality (63) holds if $\ell \mu=\hbar v$. Thus, the integral operators $\ell$ and $\hbar$ satisfy all the conditions of Theorem 7 with $\beta=b$ and $a=c=d=0$ in (5). The integral operators $\ell$ and $\hbar$ have a common fixed point, i.e., (49) has a common solution in $U$.

(c) If $N(\ell, \hbar, \mu, v)=\left(1 / t^{2}\right)\left(3 t\|\mu-v\|^{2}+\|\mu-v\|^{3}\right)$ in (55), then from (51) and (54), we have

$$
\begin{aligned}
\frac{1}{M_{r}(\ell \mu, \hbar v, t)}-1 & =\frac{d(\ell \mu, \hbar v)}{t} \leq \beta \frac{N(\ell, \hbar, \mu, v)}{t} \\
& =\beta \frac{3 t\|\mu-v\|^{2}+\|\mu-v\|^{3}}{t^{3}} .
\end{aligned}
$$

This implies

$$
\frac{1}{M_{r}(\ell \mu, \hbar v, t)}-1 \leq \beta \frac{3 t\|\mu-v\|^{2}+\|\mu-v\|^{3}}{t^{3}},
$$

for all $\mu, v \in U$ and for $t \gg \theta$. Here, we simplify the term $\left(\left(M_{r}(\mu, \ell \mu, t) * M_{r}(\nu, \hbar v, t)\right) /\left(M_{r}(\mu, v, t) * M_{r}(\mu, \hbar v\right.\right.$, $\left.\left.2 t) * M_{r}(\nu, \ell \mu, 2 t)\right)\right)-1$, by Definition 2 (3). For $t \gg \theta$, we have

$$
\begin{aligned}
\frac{M_{r}(\mu, \ell \mu, t) * M_{r}(\nu, \hbar \nu, t)}{M_{r}(\mu, v, t) * M_{r}(\mu, \hbar v, 2 t) * M_{r}(\nu, \ell \mu, 2 t)}-1 & \leq \frac{M_{r}(\mu, \ell \mu, t) * M_{r}(\nu, \hbar v, t)}{M_{r}(\mu, \nu, t) * M_{r}(\mu, v, t) * M_{r}(\nu, \hbar v, t) * M_{r}(\nu, \mu, t) * M_{r}(\mu, \ell \mu, t)}-1 \\
& =\frac{1}{M_{r}(\mu, v, t) * M_{r}(\mu, v, t) * M_{r}(\nu, \mu, t)}-1 .
\end{aligned}
$$


In view of (51) and after routine calculation, we get

$$
\begin{gathered}
\frac{M_{r}(\mu, \ell \mu, t) * M_{r}(\nu, \hbar v, t)}{M_{r}(\mu, v, t) * M_{r}(\mu, \hbar v, 2 t) * M_{r}(\nu, \ell \mu, 2 t)}-1 \\
\leq \frac{\left(3 t^{2}\|\mu-v\|+3 t\|\mu-v\|^{2}+\|\mu-v\|^{3}\right)}{t^{2}},
\end{gathered}
$$

for $t \gg \theta$. Now, from (65) and (67), we have

$$
\begin{aligned}
& \frac{1}{M_{r}(\ell \mu, \hbar v, t)}-1 \\
& \quad \leq \beta\left(\frac{M_{r}(\mu, \ell \mu, t) * M_{r}(\nu, \hbar v, t)}{M_{r}(\mu, v, t) * M_{r}(\mu, \hbar \nu, 2 t) * M_{r}(\nu, \ell \mu, 2 t)}-1\right), \quad \text { for } t \gg \theta,
\end{aligned}
$$

for all $\mu, v \in U$ such that $\ell \mu \neq \hbar v$. It is obvious that the inequality (68) holds if $\ell \mu=\hbar \nu$. Thus, the integral operators $\ell$ and $\hbar$ satisfy all the conditions of Theorem 7 with $\beta=c$ and $a=b=d=0$ in (5). The integral operators $\ell$ and $\hbar$ have a common fixed point, i.e., (49) has a common solution in $U$.

(d) If $N(\ell, \hbar, \mu, v)=\left\|\mu-A_{\mu}\right\|+\left\|v-B_{v}\right\|$ in (55), then from (51) and (54), we have

$$
\begin{aligned}
\frac{1}{M_{r}(\ell \mu, \hbar v, t)}-1 & =\frac{d(\ell \mu, \hbar v)}{t} \leq \beta \frac{N(\ell, \hbar, \mu, v)}{t} \\
& =\beta \frac{\left\|\mu-A_{\mu}\right\|+\left\|v-B_{v}\right\|}{t} \\
& =\beta\left(\frac{1}{M_{r}(\mu, \ell \mu, t)}-1+\frac{1}{M_{r}(\nu, \hbar v, t)}-1\right)
\end{aligned}
$$

This implies that

$\frac{1}{M_{r}(\ell \mu, \hbar \nu, t)}-1 \leq \beta\left(\frac{1}{M_{r}(\mu, \ell \mu, t)}-1+\frac{1}{M_{r}(\nu, \hbar v, t)}-1\right), \quad$ for $t \gg \theta$,

for all $\mu, v \in U$ such that $\ell \mu \neq \hbar v$. It is obvious that the inequality (70) holds if $\ell \mu=\hbar \nu$. Thus, the integral operators $\ell$ and $\hbar$ satisfy all the conditions of Theorem 7 with $\beta=d$ and $a=b=c=0$ in (5). The integral operators $\ell$ and $\hbar$ have a common fixed point, i.e., (49) has a common solution in $U$.

\section{Conclusion}

In this paper, we presented the concept of rational-type fuzzy cone contractions in FCM spaces and some common fixed point results under generalized rational-type fuzzy conecontraction conditions in complete FCM spaces by using the "triangular property of fuzzy cone metric" as a basic tool. Moreover, we resolved some Fredholm integral equations as an application. So, one can use this concept to prove more rational-type fuzzy cone-contraction results in complete FCM spaces with different types of applications.

\section{Data Availability}

Data sharing is not applicable to this article as no data set was generated or analysed during the current study.

\section{Conflicts of Interest}

The authors declare that there is no conflict of interest regarding the publication of this paper.

\section{Authors' Contributions}

The authors have equally contributed to the final manuscript.

\section{References}

[1] S. Banach, "Sur les opérations dans les ensembles abstraits et leur application aux équations intégrales," Fundamenta Mathematicae, vol. 3, pp. 133-181, 1922.

[2] S. K. Chatterjea, "Fixed point theorems," Dokladi na B"lgarskata Akademiya na Naukite, vol. 25, pp. 727-730, 1972.

[3] D. Chatterjea, "Geeneralized contraction principal," International Journal of Mathematics and Mathematical Sciences, vol. 6, 94 pages, 1983.

[4] H. Covitz and S. B. Nadler, "Multi-valued contraction mappings in generalized metric spaces," Israel Journal of Mathematics, vol. 8, no. 1, pp. 5-11, 1970.

[5] P. Z. Daffer and H. Kaneko, "Fixed points of generalized contractive multi-valued mappings," Journal of Mathematical Analysis and Applications, vol. 192, no. 2, pp. 655-666, 1995.

[6] E. Ameer, H. Aydi, M. Arshad, H. Alsamir, and M. Noorani, "Hybrid multivalued type contraction mappings in $\alpha \mathrm{K}$-complete partial b-metric spaces and applications," Symmetry, vol. 11, no. 1, p. 86, 2019.

[7] H. Kaneko, "Single-valued and multi-valued f-contractions," Bollettino UMI, vol. 6, pp. 29-33, 1985.

[8] R. Kannan, "Some results on fixed points," Bulletin of the Calcutta Mathematical Society, vol. 60, pp. 71-76, 1968.

[9] A. R. Khan, "Properties of fixed point set of a multivalued map," Journal of Applied Mathematics and Stochastic Analysis, vol. 2005, no. 3, pp. 323-331, 2005.

[10] K. Javed, H. Aydi, F. Uddin, and M. Arshad, "On orthogonal partial -metric spaces with an application," Journal of Mathematics, vol. 2021, Article ID 6692063, 7 pages, 2021.

[11] M. U. Ali, H. Aydi, and M. Alansari, "New generalizations of set valued interpolative Hardy-Rogers type contractions in bmetric spaces," Journal of Function Spaces, vol. 2021, Article ID 6641342, 8 pages, 2021.

[12] S. Reich, "Some remarks concerning contraction mappings," Canadian Mathematical Bulletin, vol. 14, no. 1, pp. 121-124, 1971.

[13] P. Patle, D. Patel, H. Aydi, and S. Radenović, "ON $\mathrm{H}^{+}$type multivalued contractions and applications in symmetric and probabilistic spaces," Mathematics, vol. 7, no. 2, p. 144, 2019.

[14] L. A. Zadeh, "Fuzzy sets," Information and Control, vol. 8, no. 3, pp. 338-353, 1965.

[15] O. Kramosil and J. Michalek, "Fuzzy metric and statistical metric spaces," Kybernetika, vol. 11, no. 5, pp. 336-344, 1975.

[16] A. George and P. Veeramani, "On some results in fuzzy metric spaces,” Fuzzy Sets and Systems, vol. 64, no. 3, pp. 395-399, 1994. 
[17] V. Gregori and A. Sapena, "On fixed-point theorems in fuzzy metric spaces," Fuzzy Sets and Systems, vol. 125, no. 2, pp. 245-252, 2002.

[18] M. Grabiec, "Fixed points in fuzzy metric spaces," Fuzzy Sets and Systems, vol. 27, no. 3, pp. 385-389, 1988.

[19] O. Hadzic and E. Pap, "A fixed point theorem for multivalued mappings in probabilistic metric spaces and an application in fuzzy metric spaces," Fuzzy Sets and Systems, vol. 127, no. 3, pp. 333-344, 2002.

[20] M. Imdad and J. Ali, "Some common fixed point theorems in fuzzy metric spaces," Mathematical Communications, vol. 11, pp. 153-163, 2006.

[21] F. Kiany and A. Amini-Harandi, "Fixed point and endpoint theorems for set-valued fuzzy contraction maps in fuzzy metric spaces," Fixed Point Theory and Applications, vol. 2011, no. 1, 2011.

[22] B. D. Pant and S. Chauhan, "Common fixed point theorems for two pairs of weakly compatible mappings in Menger spaces and fuzzy metric spaces," Scientific Studies and Research, vol. 21, no. 2, pp. 81-96, 2011.

[23] J. Rodriguez-Lopez and S. Romaguera, "The Hausdorff fuzzy metric on compact sets," Fuzzy Sets and Systems, vol. 147, no. 2, pp. 273-283, 2004.

[24] Z. Sadeghi, S. Vaezpour, C. Park, R. Saadati, and C. Vetro, "Set-valued mappings in partially ordered fuzzy metric spaces," Journal of Inequalities and Applications, vol. 2014, no. $1,2014$.

[25] I. Altun, B. Damjanovic, and D. Djoric, "Fixed point and common fixed point theorems on ordered cone metric spaces," Applied Mathematics Letters, vol. 23, no. 3, pp. 310-316, 2010.

[26] S. Janković, Z. Kadelburg, and S. Radenović, "On cone metric spaces: a survey," Nonlinear Analysis: Theory, Methods \& Applications, vol. 74, no. 7, pp. 2591-2601, 2011.

[27] T. Som, "Some results on common fixed point in fuzzy metric spaces, Sooc," Journal of Mathematics, vol. 33, 561 pages, 2007.

[28] L.-G. Huang and X. Zhang, "Cone metric spaces and fixed point theorems of contractive mappings," Journal of Mathematical Analysis and Applications, vol. 332, no. 2, pp. 14681476, 2007.

[29] N. B. Huy and T. D. Thanh, "Fixed point theorems and the Ulam-Hyers stability in non-Archimedean cone metric spaces," Journal of Mathematical Analysis and Applications, vol. 414, no. 1, pp. 10-20, 2014.

[30] S. Radenović, "Common fixed points under contractive conditions in cone metric spaces," Computers \& Mathematics with Applications, vol. 58, no. 6, pp. 1273-1278, 2009.

[31] M. Rangamma and K. Prudhvi, "Common fixed points under contractive conditions for three maps in cone metric spaces," Bulletin of Mathematical Analysis and Applications, vol. 4, pp. 174-180, 2012.

[32] S. U. Rehman, S. Jabeenb, and H. Ullah, "Some multi-valued contraction theorems on H-cone metric," Journal of Advanced Studies in Topology, vol. 10, no. 2, pp. 11-24, 2019.

[33] H. Aydi, E. Karapinar, and W. Shatanawi, "Coupled fixed point results for $(\psi, \varphi)$-weakly contractive condition in ordered partial metric spaces," Computers and Mathematics with Applications, vol. 62, no. 12, pp. 4449-4460, 2011.

[34] D. Turkoglu and M. Abuloha, "Cone metric spaces and fixed point theorems in diametrically contractive mappings," Acta Mathematica Sinica, English Series, vol. 26, no. 3, pp. 489496, 2010.
[35] T. Öner, M. B. Kandemir, and B. Tanay, "Fuzzy cone metric spaces," Journal of Nonlinear Sciences and Applications, vol. 8, no. 5, pp. 610-616, 2015.

[36] S. U. Rehman and H.-X. Li, "Fixed point theorems in fuzzy cone metric spaces," The Journal of Nonlinear Sciences and Applications, vol. 10, no. 11, pp. 5763-5769, 2017.

[37] A. M. Ali and G. R. Kanna, "Intuitionistic fuzzy cone metric spaces and fixed point theorems," International Journal of Mathematics And its Applications., vol. 5, pp. 25-36, 2017.

[38] G.-X. Chen, S. Jabeen, S. U. Rehman et al., "Coupled fixed point analysis in fuzzy cone metric spaces with an application to nonlinear integral equations," Advances in Difference Equations, vol. 2020, no. 1, 2020.

[39] S. Jabeen, S. U. Rehman, Z. Zheng, and W. Wei, "Weakly compatible and quasi-contraction results in fuzzy cone metric spaces with application to the Urysohn type integral equations," Advances in Difference Equations, vol. 2020, no. 1, 2020.

[40] K. Javed, F. Uddin, H. Aydi, A. Mukheimer, and M. Arshad, "Ordered-theoretic fixed point results in fuzzy b-metric spaces with an application," Journal of Mathematics, vol. 2021, Article ID 6663707, 7 pages, 2021.

[41] T. Oner, "Some topological properties of fuzzy cone metric spaces," Journal of Nonlinear Sciences and Applications, vol. 9, no. 3, pp. 799-805, 2016.

[42] T. Oner, "On some results in fuzzy cone metric spaces," International Journal of Advance Computational Engineering and Networking., vol. 4, pp. 37-39, 2016.

[43] T. Oner, "On the metrizability of fuzzy cone metric spaces," International Journal of Management and Applied Science., vol. 2, pp. 133-135, 2016.

[44] S. U. Rehman, Y. Li, S. Jabeen, and T. Mahmood, "Common fixed point theorems for a pair of self-mappings in fuzzy cone metric spaces," Abstract and Applied Analysis, vol. 2019, Article ID 2841606, 10 pages, 2019.

[45] S. U. Rehman, I. Khan, H. Ullah, S. Jabeen, and F. Abbas, "Common fixed point theorems for compatible and weakly compatible maps in fuzzy cone metric spaces," Annals of Fuzzy Mathematics and Informatics, vol. 19, no. 1, pp. 1-19, 2020.

[46] N. Priyobarta, Y. Rohen, and B. B. Upadhyay, "Some fixed point results in fuzzy cone metric spaces," International Journal of Pure and Apllied Mathematics, vol. 109, no. 3, pp. 573-582, 2016.

[47] B. Schweizer and A. Sklar, "Statistical metric spaces," Pacific Journal of Mathematics, vol. 10, no. 1, pp. 313-334, 1960. 